\title{
A Reduced Role of V/Q Scintigraphy in the Diagnosis of Acute Pulmonary Embolism
}

V entilation/perfusion (V/Q) lung scintigraphy, when used for the clinical determination of the presence or absence of pulmonary embolism, has been much studied by medical imagers. Inherent to a "cold-spot" scan targeting the normal pulmonary tissue, the deck has always been stacked against us and our specialty frequently maligned by the referring physicians as practicing "unclear" and not nuclear medicine. The nonspecificity and, at times reputed, nonsensitivity of the V/Q scan for embolism as imaging protocols evolved led to a probability scheme (I) of reporting positive or negative findings in shades of gray rather than as

\section{See page 1411}

a definitive black-and-white clinical statement. Given that pulmonary embolism is so frequent ( $>500,000$ annually in the United States), and severe (the third leading cause of death in the United States, with a fatality rate of approximately $30 \%$ from each event) (2), and given that nonspecific clinical signs and symptoms lead to frequent misdiagnosis at presentation, referring clinicians increasingly have sought alternative noninvasive diagnostic means.

PIOPED I (Prospective Investigation of Pulmonary Embolism Diagnosis I) was our first effort to provide clarity (3). Coupling V/Q scintigraphy and chest radiography to invasive contrast pul-

Received May 17, 2007; revision accepted Jun. 27, 2007.

For correspondence or reprints contact: Arnold M. Strashun, MD, Nuclear Medicine Department, SUNY Downstate Medical Center, 450 Clarkson Ave., Brooklyn, NY 11203.

E-mail: astrashun@downstate.edu

COPYRIGHT @ 2007 by the Society of Nuclear Medicine, Inc.

DOI: 10.2967/jnumed.107.041988 monary angiography, this large multicenter trial demonstrated in 1990 a wide range of diagnostic uncertainty in the scintigraphic diagnosis of acute embolism. The prevalence of acute embolic disease within a particular referral basewhich could vary from $25 \%$ of all studied to as high as $50 \%$, with an average of $35 \%$ in most published studies-was found to strongly affect the final prediction of certainty of embolism. For example, low prevalence of disease populations with a single segmental V/Q mismatch could have a $30 \%$ likelihood of acute embolism as compared with a $70 \%$ likelihood with the same scintigraphic findings in a high prevalence group. This study did provide extensive patient data, which subsequently led to revised V/Q scan criteria and better precision in predicting angiographyconfirmed acute embolism $(4,5)$.

In the binary age of the computer, clinicians continued to search for a yes/ no noninvasive test for acute pulmonary embolism. CT has rapidly evolved to fill this role. CT detector technology advanced from single-detector spiral scanners to multidetector CT systems with powerful computers and volumetric reconstruction algorithms capable of virtual real-time pulmonary angiography. Multidetector CT angiography (MDCTA) changed a slow, crude, and unreliable study of central pulmonary arterial anatomy to a fast and anatomically precise determinant of the integrity of the smallest caliber pulmonary arterial branches. Of course, this evolution has come at a cost in dollars and radiation burden. Most clinicians prefer this cost to the alternative imprecision and diagnostic uncertainty of V/Q scintigraphy. Accordingly, most medical centers with MDCTA capability have all but abandoned the V/Q scan for the initial diagnosis and follow-up of pulmonary embolism, even before definitive evidence-based comparative data of this new technology were analyzed and published. As important, two thirds of patients with acute symptoms studied with MDCTA to exclude embolism yield an alternative, definitive anatomic diagnosis other than pulmonary embolism, which is not generally possible with V/Q scintigraphy findings referable only to the pulmonary vasculature (6).

PIOPED II, the prospective multicenter study funded by the National Heart, Lung, and Blood Institute and composed of expert imagers and clinicans, began recruiting patients in 2001 to determine the relative value of current clinical bedside, laboratory, and imaging data, including MDCTA in the diagnosis of acute pulmonary embolism. After a decade of study, these investigators in landmark publications $(7,8)$ show that clinicians' clinical preimaging probability assessment is codifiable and a reliable predictor of definitive imaging confirmation of acute embolism. D-dimer enzymelinked immunosorbent assays are critical to this stratification-that is, less than a high (moderate or low) clinical pretest probability for pulmonary embolism and a negative $\mathrm{D}$-dimer assay do not justify any MDCTA or scintigraphy. If otherwise, MDCTA is the recommended first imaging procedure. MDCTA outcomes data support this advice. First, MDCTA for embolism is reliable in most hands, with $94 \%$ (773/ 824) satisfactory quality in acute pulmonary embolism evaluation. Second, MDCTA is very sensitive, with a positive predictive value for acute pulmonary embolism of $86 \%$ (150/175). This sensitivity declines directly with severity - that is, physiologically more catastrophic central emboli are seen 
with MDCTA in 97\% (116/120) of afflicted patients. Segmental-only defects were detectable by MDCTA in 68\% (32/47), and the very rare subsegmentalonly emboli were detected in only $25 \%(2 / 8)$. It is important to note that the majority of MDCTA devices in PIOPED II used only 4,8 , or 16 detectors and that resolution of smaller emboli should definitely be improved with newer systems. Third, the specificity of MDCTA is even higher, as expected, with a negative predictive value of $95 \%$ (567/598). These data translate into an acute pulmonary embolism likelihood ratio with a positive MDCTA of 19.6 versus 0.2 with a negative study. MDCTA clearly exceeds the V/Q scan at both ends of the clinical probability spectrum, and its technical reliability and additional nonembolic findings eliminate the need for scintigraphy, even in the middle probability ground in most clinical situations. Finally, CT venography, when added to the MDCTA protocol, further lowers the likelihood ratio of a negative CTA for embolism to 0.1. However, CT venography does significantly raise the radiation burden from a range of 1.6-8.3 $\mathrm{mSv}$ with varying MDCT pulmonary angiography protocols by an additional estimated $5.7 \mathrm{mSv}$. This radiation exposure should be contrasted with a standard posteroanterior and lateral chest radiograph at $0.07 \mathrm{mSv}$ and a standard V/Q scan at 1.2-2.0 $\mathrm{mSv}$ (7).

Given these data, there is very little room left for performing V/Q scintigraphy in the diagnosis of acute pulmonary embolism were it not for 2 major risks inherent to current contrast MDCTA. First, all contrast media, whether ionic/nonionic or hyper/ hypoosmolar, have inherent and significant risks. In addition to a wide range of frequent allergic reactions, contrast-induced renal damage risk is severe and proportional to the level of renal dysfunction frequently seen in patients at higher risk for acute pulmonary embolism (the sedentary, elderly, postoperative, congestive heart failure, pregnant, etc.). A rule of thumb is that a glomerular filtration rate of $<30$ is a contraindication to contrast
MDCTA, as the risk of contrast nephropathy at that preexisting level of renal dysfunction is clinically unacceptable in almost every circumstance. Second, MDCTA produces significant radiation burden, which leads to statistically predictable risks of mutagenicity to at-risk tissue irradiated. This is especially true for the female breast during and before the reproductive years. Worst-case estimates suggest that the typical MDCTA for pulmonary angiography delivers as much as a 50-mGy absorbed dose to the breast (as compared with 0.28 mGy with a perfusion scan) $(9,10)$. The additional lifetime risk of radiationinduced breast cancer from such a single MDCTA exposure could be in the range of as much as 1 in 500 to as little as 1 in 5,000 (currently the overall preexisting risk of breast cancer for U.S. women is as much as 1 in 8) $(11,12)$.

For these reasons, Gottschalk et al. (13), in this issue of The Journal of Nuclear Medicine, revisited their PIOPED II data and found that the use of their specific standardized multifactorial clinical criteria yielding a low positive predictive value, together with the use of strictly graded very low probability V/Q scans, proved as reliable as a negative MDCTA, with a negative predictive value of $97 \%$ (251/259) of a confirmatory negative digital subtraction angiogram as gold standard to exclude the diagnosis of acute pulmonary embolism. This is important as the majority of referrals do ultimately prove negative for acute embolism, with a range of 50\%-75\% and, on average, $70 \%$ in most published studies. A very low probability V/Q result is a frequent finding: $56 \%$ of all patients in PIOPED II. Thus, the authors conclude from this analysis that women of reproductive age should first undergo a V/Q scan rather than MDCTA if their clinical pretest likelihood of pulmonary embolism as graded by an experienced clinician is low, as they are likely to have pulmonary embolism excluded by scintigraphy with the same certainty as MDCTA but with less potential radiation risk. In addition, patients with severe contrast allergy history and those with compromised renal function should also first undergo a V/Q scan if contrast-protective regimens cannot first be applied (13). In consideration of acute pulmonary embolism, the V/Q scan in the majority of patients with these few exceptions is no longer the pivotal diagnostic imaging study in 2007.

In conclusion, the evolution of more precise chemical and clinical screening for pulmonary thromboembolism coupled with diagnostic refinements in multidetector CT produces a clearer and diagnostically more useful determination than scintigraphy of the presence or absence of pulmonary embolism. Were it not for definite allergic and nephrotoxic risks of contrast media and the added radiation burden of MDCTA, the ventilation/perfusion scan would virtually disappear from the diagnostic algorithm for pulmonary embolism.

Arnold M. Strashun
SUNY Downstate Medical Center
Brooklyn, New York

\section{REFERENCES}

1. Biello DR, Mattar AG, McNight RC, Siegel BA. Ventilation-perfusion studies in suspected pulmonary embolism. AJR. 1979;133:1033-1037.

2. Dalen JE, Alpert JS. Natural history of pulmonary embolism. Prog Cardiovasc Dis. 1975;17:259270.

3. The PIOPED Investigators. Value of the ventilation/perfusion scan in acute pulmonary embolism: results of the Prospective Investigation of Pulmonary Embolism Diagnosis (PIOPED). JAMA. 1990; 263:2753-2759.

4. Gottschalk A, Sostman HD, Coleman RE, et al. Ventilation-perfusion scintigraphy in the PIOPED study. Part II. Evaluation of the scintigraphic criteria and interpretations. J Nucl Med. 1993;34: 1119-1126.

5. Worsley DF, Alavi A. Comprehensive analysis of the results of the PIOPED study. J Nucl Med. 1995;36:2380-2387.

6. Schoepf UJ, Castello P. CT angiography for diagnosis of pulmonary embolism: state of art. Radiology. 2004;230:329-337.

7. Stein PD, Woodward PK, Weg JG, et al. Diagnostic pathways in acute pulmonary embolism: recommendations of the PIOPED II investigators. Radiology. 2007;242:15-21.

8. Stein PD, Fowler SE, Goodman LR, et al. Multidetector computed tomography for acute pulmonary embolism. N Eng J Med. 2006;354: 2317-2327. 
9. Cook JV, Kyriou J. Radiation from CT and perfusion scanning in pregnancy. BMJ. 2005;331:350.

10. Parker MS, Hui FK, Camacho MA, Chung JK, Broga DW, Sethi N. Female breast radiation exposure during CT pulmonary angiography. AJR. 2005;185:1228-1233.
11. Allen C, Demetriades T. Radiation risk overestimated. Radiology. 2006;240:613-614.

12. National Research Council of the National Academies. Health Risks from Exposures to Low Levels of Ionizing Radiation. BEIR VII. Washington, DC: The National Academies Press; 2005.
13. Gottschalk A, Stein PD, Sostman HD, Matta F, Beemath A. Very low probability interpretation of V/Q lung scans in combination with low probability objective clinical assessment reliably excludes pulmonary embolism: data from PIOPED II. J Nucl Med. 2007;48:1411-1415. 